\title{
MODELING METALLURGICAL SUPPLY CHAIN RESILIENCE USING MARKOV PROCESS
}

\author{
${ }^{1}$ Martin ČECH, ${ }^{1}$ Radim LENORT, ${ }^{1}$ Pavel WICHER, ${ }^{2}$ Tatiana TOLSTYKH, ${ }^{2}$ Elena SHKARUPETA \\ ${ }^{1}$ VSB - Technical University of Ostrava, Faculty Ostrava, Czech Republic, EU, martin.cech@vsb.cz \\ 2Institute of Economics and Industrial Management, The National University of Science and Technology \\ "MISIS", Moscow, Russian Federation, nshmeleva@misis.ru
}

https://doi.org/10.37904/metal.2019.782

\begin{abstract}
Metallurgical supply chain consists of raw materials miners, transport, integrated metallurgical enterprise, service centers and local distributors. The overall performance of the supply chain which is subject to disruption from the environment is dependent on performance of its links and its ability to recovery after such event or resilience, respectively. As it is possible to estimate mean time between failures and mean time to recovery for each supply chain link, the whole process can be modeled as a stochastic process using Markov process. This article aims in setting assumptions for a model, creating a model of metallurgical supply chain in the context of supply chain resilience using Markov process which would allow to asses resilience using overall supply chain performance as an indicator.
\end{abstract}

Keywords: Metallurgy, modeling, supply chain, resilience, Markov process

\section{INTRODUCTION}

Among the most important approaches to the need for the evolution of the concept of leanness that is difficult to operate in today's market environment is the concept of agility [1], [2], which has been incorporated into the concept of resilience based on current research in this area [3]. When defining supply chain resilience, the meaning of the latin resiliere - the ability of a substance or object to return to its original form (flexibility) or its ability to recover quickly from difficulty (toughness) can be taken. One of the most significant contemporary authors defining resilience is Christopher and Rutherford [4], who places particular emphasis on the flexibility and adaptability of the system. Fiksel [5] defines resilience as "the ability to survive, adapt and grow despite turbulent changes in the environment". For the purposes of this article, the definition presented by Sheffi and Rice [6] can be used: "resilience is the ability to return from a disrupted state.

Although supply chain resilience assessment has been studied by a number of authors, the issue of supply chain resilience assessment and building is still in its infancy, and concrete tools to support decision-making in supply chain resilience have not yet been presented. The main issue motivating this study is: To which links of the metallurgical supply chain and to what extent to allocate funds to increase resilience so that the resulting effect is as high as possible? The goal of the article can then be divided into 2 sub-goals:

- Designing a mathematical model of supply chain to assess the impact of funds allocated to increase the supply chain's resilience to its overall performance.

- Developing experimental application to model allocation of funds and its effects allowing a future implementation of optimization algorithm.

\section{SUPPLY CHAIN RESILIENCE AND DISRUPTIONS}

Resilience is seen as the ability of the supply chain to return to its original state after an unexpected disruption. The process of changing supply chain performance before, during and after disruption can be characterized by 8 phases - preparatory phase, incidence of disruption, first response, initial impact, full impact moment, recovery preparation, recovery, long-term impact. 
The basic ways to achieve supply chain resilience include building overcapacity and flexibility, building the ability to move production between plants, leveraging parallel product development processes, designing products and processes to maximize product de-completion, or even crucial relationship development with suppliers [7]. Based on current literature, building on the supply chain resilience can also be approached through the improvement of specific skills (according to [6], [7], [8], [9], [10], [11], [12]). Supply chain disruption means a reduction in functionality, resp. performance or the complete decommissioning of individual elements or the entire supply chain as a result of an internal or external event. According to the World Economic Forum, main sources of disruption can be defined - natural disasters and extreme weather, political conflicts and problems or terrorism, sudden radical changes in demand.

\section{MODELING ASSUMTIONS}

For the purposes of modeling set of assumptions in three categories was defined considering logistics, methodological and economic aspects of the proposed model. Due to the limited page range of the article only the main assumptions are presented here:

\subsection{Logistics assumptions}

- $\quad$ The supply chain is capacity balanced and cannot be blocked by products pending.

- The rate of occurrence of disturbances and their duration are exponential random variables.

- $\quad$ For each supply chain link, the mean time between failure (MTBF) and the mean time to recovery (MTTR) may be determined.

- SC resilience can be increased by allocating funds, which will lead to shortening the duration of the disturbances.

\subsection{Methodological assumptions}

- Supply chain performance can be estimated on the basis of stabilized probabilities of occurrence of the system in individual possible states calculated on the basis of the Markov process theory.

- Due to the use of the discrete-time Markov process theory, the mean duration of the disruption (until article recovery) can be reduced to a maximum of 1 . In case of reduction to less than 1 resp. at 0 , the article will behave as "trouble-free", which has a major impact on the construction of the whole task.

- The time dimension will be implicitly included in the model as a parameter of maximum supply chain performance.

\subsection{Economic assumptions}

- $\quad$ Supply chain performance is represented by the accumulated undiscounted cash flow.

- $\quad$ Funds invested in the modeled period to improve the supply chain (to increase its resilience) are seen as a simple investment expense without discounting.

\section{MODEL}

The outcome of the mathematical model is assumed to be the supply chain response to the specific distribution of available funds, designed to increase the resilience of the supply chain, among its individual links. On the basis of the economic and logistical assumptions set out in the previous chapter, the supply chain response to the needs of the article means an absolute increase in the performance of the supply chain in question in the modeled period due to the allocation of funds to increase the resilience of its individual links. This increase in performance of supply chain as a whole is compared with performance without increasing resilience, respectively without spending money to increase resilience. Let us call the "scenario" a specific scheme of 
allocating funds among the links in the supply chain. Then, in determining the response value for the different allocation funds scenarios, these scenarios can be compared and the most favorable one selected.

The most advantageous allocation scenario is the one in which the allocation of available funds brings the supply chain as a whole the highest economic effect, respectively the scenario that delivers the greatest supply chain response. The basic idea is thus expressed by the general formula for calculating supply chain response $\mathrm{O}_{i}$ :

$$
O_{I}=V_{I}-V_{0}-\sum_{i=1}^{n} I_{i},
$$

where:

$O_{I} \quad$ - supply chain response to a specific allocation scheme between individual supply chain links (EUR),

$V_{I} \quad$ - supply chain performance in the modeled period with a specific allocation scheme, resp. after increased resilience, so-called improved performance (EUR),

$V_{0} \quad$ - supply chain performance in the modeled period without allocating funds to increase resilience, socalled original performance (EUR),

$I_{i} \quad$ - the amount of funding allocated to increase the resilience of the $i$-th supply chain article (EUR),

I $\quad-I=\left(I_{1}, I_{2}, \ldots, I_{n}\right)$ a specific scheme of allocation of funds between individual links in the supply chain, the so-called "scenario" (EUR)

$I_{0} \quad-I_{0}=(0,0, \ldots, 0)$ a scenario where no increase in resilience is made (with no allocation),

$n \quad$ - number of supply chain links $(i=1,2, \ldots, n)$.

Partial calculations are required to determine the supply chain response. The calculation of the total amount of funds allocated to increase the supply chain resilience $\sum_{i=1}^{n} I_{i}$ is a simple sum of all partial allocations of funds into individual articles. The following conditions must be met based on the assumptions:

$$
\sum_{i=1}^{n} I_{i} \in\left\langle 0 ; I_{\max }\right\rangle,
$$

where $I_{\max }$ is the maximum sum of all investments.

To determine the performance of the supply chain after increasing its resilience by allocating funds at a particular level of $I_{i}$ for individual links (hereinafter referred to as "improved performance"), respectively its performance without the allocation of funds (hereinafter "original performance"), based on the assumptions of the model, the representation of the behavior of the supply chain under consideration by Markov chain is used.

Supply chain behavior can be seen as a Markov process, a sequence of integer random variables $\left\{X_{\tau}, \tau \in \mathbb{N}\right\}$ on the probability space. Let $S$ be the finite set of random process states $\left\{X_{\tau-1}, \tau \in \mathbb{N}\right\}$ and its elements be states of this process. For each supply chain link, there are 2 states in which it can be found over time:

- $\quad i$-th link is in full operation,

- $\quad i$-th the article is under the influence of disruption.

As described above, in the modeled supply chain, for each of its articles operating in a different region, the linear dependence of the mean recovery time (disruption duration) on the amount of funding allocated to increase the resilience of that article applies. This dependency is considered and defined for each article only on the interval $\left\langle I_{i, \min } ; I_{i, \max }\right\rangle$, where $i=1 \ldots n$, resp. the number of supply chain links is $n=2$. This condition reflects the fact that the resilience of individual articles, or the entire supply chain, starts to increase from a certain volume of allocated funds. Therefore, as long as at least the minimum amount of funds required (input 
parameter $I_{i, \min }$ ) is not invested in the $i$-th supply chain link, its resilience remains the same and the $M T T R_{i}$ does not change. Using the general straight-line equation, it is possible for $M T T R_{i}$, resp. mean time to recovery (disruption duration) $i$-th supply chain link when certain amount of funds $I_{i}$ is allocated, derive the equation using previously defined model input parameters:

$$
\operatorname{MTTR}_{i}=\left\{\begin{array}{cl}
M T T R_{i, 0} & - \text { if } I_{i} \in\left\langle 0, I_{i, \text { min }}\right\rangle, \\
\frac{M T T R_{i, 0} \cdot\left(I_{i}-I_{i, \text { max }}\right)}{I_{i, \min }-I_{i, \text { max }}} & - \text { if } I_{i} \in\left\langle I_{i, \text { min }}, I_{i, \max }\right\rangle, \\
0 & - \text { if } I_{i}>I_{i, \text { max }},
\end{array}\right.
$$

where:

$I_{i} \quad$ - investment allocated to increase the resilience of the $i$-th supply chain link,

$I_{i, \max }$ - the amount of investment needed to fully eliminate the impacts of disruption of the $i$-th supply chain link,

$I_{i, \min }$ - the minimum amount of investment that begins to increase resilience, which will translate into an increase in the resilience of the $i$-th supply chain article,

$M T T R_{i, 0}$ - Mean Time to Recovery, if not invested in $i$-th supply chain links resilience improvement.

The relationship defined above only makes sense in the context of the studied problem and the modeled supply chain when the following conditions are met:

$$
\begin{gathered}
0 \leq I_{i, M I N} \leq I_{i}<I_{i, M A X}, \\
M T T R_{i, 0}>0 .
\end{gathered}
$$

For each supply chain link it is now possible to determine the frequency of restoration $\mu_{i}$, respectively, the probability of transition from a state under the influence of a disruption " 0 " to a full power state " 1 ", respectively $\lambda_{i}$, the probability of transition from full power state "1" to reduced power state under the influence of disruption "0":

$$
\begin{aligned}
& \mu_{i}=\frac{1}{M T T R_{i}}, \\
& \lambda_{i}=\frac{1}{M T B F_{i}} .
\end{aligned}
$$

From the logic of the behavior of the modeled supply chain and the above-described states in which individual links may be, respectively the entire supply chain can occur, then the probability of $\mu_{i}^{\prime}$ with which the $i$-th link under the influence of the disruption will remain disrupted when the supply chain moves to the next state and vice versa $\left(\lambda_{i}^{\prime},\right)$ can be deduced for each link:

$$
\begin{aligned}
& \mu_{i}^{\prime}=1-\mu_{i}=1-\frac{1}{M T T R_{i}}, \\
& \lambda_{i}^{\prime}=1-\lambda_{i}=1-\frac{1}{M T B F_{i}} .
\end{aligned}
$$

Above-derived formulas for the probability of changing the status of individual cells when changing the supply chain state are further used to compile the formulas for calculating the transition probabilities, needed to construct the probability transition matrix of the Markov chain $\boldsymbol{P}$. Matrix $\boldsymbol{P}$ of the probabilities of transitions made up of the above derived formulas then takes the form: 


$$
\boldsymbol{P}=\left(\begin{array}{ccc}
p_{00} & \cdots & p_{03} \\
\vdots & \ddots & \vdots \\
p_{30} & \cdots & p_{33}
\end{array}\right) .
$$

After compilation of the matrix of probabilities of transition $\boldsymbol{P}$ the system of equations can be solved based on the Markov chain theory:

$$
\begin{aligned}
& \boldsymbol{a} \cdot \boldsymbol{P}=\boldsymbol{a} \\
& \sum_{s=0}^{3} a_{s}=1,
\end{aligned}
$$

Where:

$$
\begin{array}{ll}
\boldsymbol{P} & \text { - is a matrix of transitions between states compiled from the above relationships, } \\
\boldsymbol{a} & \text { - is a stationary vector of probabilities of occurrence of supply chain states, while } \boldsymbol{a}=\left\{a_{s}\right\}_{s=0}^{3}, \\
a_{s} & - \text { is a stationary probability of individual supply chain states for } s=0, \ldots, 3
\end{array}
$$

For individual supply chain states, using the previously calculated $a_{s}$ probabilities of occurrence in individual states, input parameters $V_{1, N O K}$ and $V_{2, N O K}$ indicating the relative performance of individual cells under the influence of a disruption and $V_{\max }$ indicating the maximum possible performance of the supply chain, can be used to calculate $V_{I}^{s}$ supply chain performance in each state. This $V_{I}^{s}$ is determined as the product of the supply chain probability of occurrence of the supply chain in the given state $s$, the lowest $V_{i, N O K}$ relative performance of the cell if at least one of the supply chain cells is affected by the disruption, and $V_{\max }$, the maximum supply chain performance in period without disturbance.

Anaconda Navigator user interface was used to implement the model as well as interactive development environment Spyder, enabling creation, editing and execution of created programs. Libraries SciPy, NumPy and Pandas were used to create a program based on the above-described mathematical model and verbal description of its calculation algorithm. Algorithm to calculate a mathematical model, which is the basis for creating a program in a Python environment that allows experiments on the model is as follows:

- Define supply chain structure, enter the number of supply chain layers and assign links to layers.

- $\quad$ Enter input parameters for each supply chain link and entering the maximum possible supply chain performance and maximum volume of possible investment.

- $\quad$ Generate a specific investment scheme.

- $\quad$ Calculate cell transition probabilities between full traffic and violation states.

- $\quad$ Generate all possible supply chain states and calculate of the supply chain probability matrix.

- $\quad$ Calculate stationary vector probability of supply chain occurrence in individual states.

- $\quad$ Calculate supply chain performance for a particular investment scheme being tested.

- Determine the supply chain response to a specific investment scheme allocated to increased resilience.

\section{CONCLUSION}

Using Markov chain allows to model funds allocation in supply chain resilience improvements under certain set of initial assumptions, which has to be considered before making real investment decision. Markov chain is not difficult methodology with a quite simple mathematical algorithm. General model for SC of more layers and links, thus various structures, implemented in Python was developed. The developed model and experimental application allow to compare allocation scenarios and can be used as a decision support tool in the process of SCM and resilience improvements. Developed tool is relatively simple and easy to implement 
and use compare to production line models and represents a novel approach to modeling in the field of supply chain resilience.

\section{ACKNOWLEDGEMENTS}

The work was supported by the specific university research of the Ministry of Education, Youth and Sports of the Czech Republic in VSB - Technical University of Ostrava No. SP2019/42 and SP2019/62.

\section{REFERENCES}

[1] RIMIENE, K. Supply chain agility concept evolution (1990-2010), EKONOMIKA IR VADYBA, vol. 16, 2011, pp. 892-899.

[2] SHARIFI, H., ZHANG, Z. A methodology for achieving agility in manufacturing organisations: An introduction. International Journal of Production Economics, vol. 62, no. 1-2, 1999, pp. 7-22.

[3] LENORT, R., WICHER, P. Agile Versus Resilient Supply Chains: Commonalities and Differences. In CLC 2012: Carpathian Logistics Congress. [CD-ROM] Ostrava: TANGER, 2012, pp. 558-564.

[4] CHRISTOPHER, M., RUTHERFORD, C. Creating Supply Chain Resilience through Agile Six Sigma. Critical Eye. no. June - August 2004, pp. 24-28.

[5] FIKSEL, J. Sustainability and Resilience: Towards a System Approach. Sustainability: Science, Practice \& Policy, vol. 2, no. 2, 2006, pp. 1-8.

[6] SHEFFI, Y. A Supply Chain View of the Resilient Enterprise. USA: MIT Sloan, vol. 47 no. 1, 2005, pp. 8-41.

[7] SHEFFI, Y. et al. Supply Chain Resilience How Can You Transcend Vulnerability In Your Supply Chain to Gain Competitive Advantage? The Official Magazine of the Logistics Institute. vol. 12, no. 1, 2006.

[8] CARVALHO, H., MACHADO, V.C. Designing principles to create resilient Supply Chains. In Proceedings of the 2007 Industrial Engineering Research Conference, Caracas: Catholic University. Andres Bello, G. Bayraksan, W. Lin, Y. Son, and R. Wysk, eds. 2007.

[9] ENYINDA, C.I., SZMEREKOVSKY, J. Sense and Respond Supply Chain: A Prescription for Mitigating Vulnerability in the U.S. Pharmaceutical Value Chain. Journal of Global Business Issues. vol. 2, no. 2, 2008, pp. 95.

[10] FALASCA, M. et al. A Decision Support Framework to Assess Supply Chain Resilience. In Proceedings of the 5th International ISCRAM Conference, Washington DC, USA, May 2008 F. Fiedrich and B. Van de Walle, eds. 2008.

[11] IAKOVOU, E., VLACHOS, D., XANTHOPOULOS, A. An analytical methodological framework for the optimal design of resilient supply chains. International Journal of Logistics Economics and Globalisation. vol. 1, 2007, pp. 1-20.

[12] TANG, C.S. Robust strategies for mitigating supply chain disruptions. International Journal of Logistics: Research and Applications. vol. 9, no. 1, 2006, pp. 33-45. 